\title{
Enhancing wuyiencin productivity of Streptomyces albulus (CK15) by mutagenesis breeding with atmospheric and room temperature plasma
}

\section{Yu Wah Thein}

Mandalay Technological University

\section{Liming Shi}

Chinese Academy of Agricultural Sciences

Binghua Liu

Chinese Academy of Agricultural Sciences

\section{Qiuhe Wei}

Chinese Academy of Agricultural Sciences

\section{Kecheng Zhang}

Chinese Academy of Agricultural Sciences

\section{Beibei Ge ( $\nabla$ bbge@ippcaas.cn )}

Chinese Academy of Agricultural Sciences Institute of Plant Protection https://orcid.org/0000-00027365-9872

\section{Research Article}

Keywords: Streptomyces albulus, wuyiencin, atmospheric and room temperature plasma (ARTP)

Posted Date: February 10th, 2022

DOI: https://doi.org/10.21203/rs.3.rs-1288176/v1

License: (9) This work is licensed under a Creative Commons Attribution 4.0 International License. Read Full License 


\section{Abstract}

Streptomyces species are commonly used for efficient secondary metabolite production, including many antibiotics. Wuyiencin is a secondary metabolite produced by Streptomyces albulus CK15. This antibiotic is used in agriculture for controlling various fungal diseases of vegetables and field crops. In the present study, we used atmospheric and room temperature plasma (ARTP) mutagenesis to generate mutant $S$. albulus strains with the capacity for improved fermentation production of wuyiencin. The wild-type $S$. albulus CK15 strain was mutagenized by ARTP for one time and screened for two rounds of antimicrobial activity, three genetically stable mutants (M19, M26, and M28) were identified, which showed increased wuyiencin production by $17.41 \%, 13.57 \%$ and $18.46 \%$ compared to the CK 15 strain in flask culture, respectively. The M28 mutant exhibited a maximum wuyiencin activity of $1442.95 \pm 134.61 \mathrm{U} / \mathrm{mL}$ in flask culture and $1673.84 \pm 127.42 \mathrm{U} / \mathrm{mL}$ in a $5 \mathrm{~L}$ fermenter. Therefore, our results provide evidence that ARTP is an efficient tool for microbial mutation breeding and the enhancement of wuyiencin production.

\section{Introduction}

Streptomyces are Gram-positive bacteria that grow in various environments, with a shape that resembles filamentous fungi. Streptomyces species are important due to their ability to produce bioactive secondary metabolites such as antifungals, antivirals, antineoplastics, and antihypertensives, but mainly antibiotics and immunosuppressives (Khan et al. 2011; Ōmura et al. 2001; Patzer and Braun 2010). More than 500 species of the genus Streptomyces have been described and nearly two-thirds of all naturally occurring antibiotics are produced by S. species (Mohanraj and Sekar 2013). Streptomyces albulus var. wuyiensis CK15 was isolated from a soil sample collected from the Wuyi Mountains in Fujian Province, China. This strain produces the antibiotic wuyiencin, which has wide applications in agriculture for the control of various fungal diseases of vegetables and field crops (Cui et al. 2010). Wuyiencin is characterized by high efficiency and low toxicity compared with that of other chemical pesticides. Wuyiencin products ( $1 \%$, $2 \%$ and $3 \%$ ) are widely used as biopesticides recommended for pollution-free vegetable production in China (Ge et al. 2015). However, the lower yield and higher cost of wuyiencin restricts its further largescale application. Therefore, modified CK15 strains exhibiting high wuyiencin productivity are required for long-term use. In previous studies, although high-yield strains were breeded by physical and chemical mutagenesis, no good high-yielding strains were obtained. Recently, various efforts have been made to improve wuyiencin yield through genetic manipulation. For example, marked increases in wuyiencin yield were achieved by overexpression of wysR, a key gene involved in wuyiencin biosynthesis (Liu et al. 2014).

Microbial mutation breeding by altering the genomes shows great potential for biotechnology research and applications (Kumar et al. 2015; Tan et al. 2014). Microbial breeding mutagenesis can be achieved using various approaches, mainly physical, chemical and biological methods (Guo 2018). Both traditional and newly developed mutagenesis methods such as atmospheric and room temperature plasma (ARTP) represent an effective and rapid way to enhance the production of secondary metabolites (Sivaramakrishnan and Incharoensakdi 2017; Zhang et al. 2016). Recently, ARTP was developed as a novel and efficient mutation tool that has been successfully employed to generate significant changes in 
enzyme activity, biochemical productivity and metabolism, without lethal damage (Liu et al. 2020; Gu et al. 2020; Qiu et al. 2021; Lu et al. 2011; Qiang et al. 2014; Xu et al. 2012). The core component of ARTP is Radio Frequency Atmospheric Pressure Glow Discharge(RF APGD)plasma generator, which generates a plasma jet that can change structure and permeability of the cell wall and plasma membrane, leading to DNA damage, including missense mutation, deletion or nucleotide frame shift mutation (Fang et al. 2013; Li et al. 2014; Zhang et al. 2014). Atmospheric pressure glow discharge (APGD) plasma sources are becoming increasingly popular in various fields, such as plasma-based sterilization, disinfection, and industrial microbial mutations (Li et al. 2008). This is due to the low and controllable gas and temperature they require, the high concentrations of the chemically active species, and their flexible operation (Wang et al. 2010; Laroussi 2005).

Compared to conventional mutation breeding methods, ARTP mutagenesis has many advantages. The procedure is simple, not harmful to humans and causes no environmental pollution. Furthermore, the mutagenic temperature is room temperature, and the process is carried out under normal pressure. The active particle composition in the jet is diverse and the concentration is high. The biological damage mechanism and mutational diversity are especially suitable for microorganisms with complex metabolic networks (Wang et al. 2020; Guo 2018). The features of ARTP technology are suitable for improved breeding of spore-forming bacteria. The ARTP system has been successfully applied in $S$. species, such as Streptomyces avermitilis and Streptomyces bingchenggensis, to improve avermectin and milbemycins A3/A4 production levels, respectively (Wang et al. 2014; Wang et al. 2010). In order to obtain a desired product, a single ARTP treatment or an iterative ARTP treatment is usually performed (Wang et al. 2010; Ren et al. 2017; Cao et al. 2018). In this study, we applied the ARTP technique to enhance wuyiencin productivity in $S$. albulus.

\section{Materials And Method \\ 2.1. Strains and media}

The wild-type $S$. albulus CK15 strain (stored in our laboratory) and the mutant strains generated by ARTP mutagenesis were cultured at $28^{\circ} \mathrm{C}$ on mannitol-soybean (MS) agar or in yeast extract malt extract (YEME) liquid medium. The soluble fermentation medium consisted of $2 \mathrm{~g}$ soybean flour, $2 \mathrm{~g}$ glucose, $3 \mathrm{~g}$ corn starch, $300 \mathrm{mg} \mathrm{CaCO}_{3}, 400 \mathrm{mg}\left(\mathrm{NH}_{4}\right)_{2} \mathrm{SO}_{4}$, dissolved in $\mathrm{H}_{2} \mathrm{O}$ to give a final volume of $100 \mathrm{~mL}$. Rhodotorula rubra was incubated in potato dextrose agar (PDA) medium at $28^{\circ} \mathrm{C}$ overnight and was used as an indicator strain in the wuyiencin bioassays. All the media were autoclaved at $121^{\circ} \mathrm{C}$ for $30 \mathrm{~min}$.

\subsection{ARTP mutagenesis method}

The procedures for mutation of $S$. albulus using the ARTP breeding system were conducted at the Chemical Engineering Department, Tsing Hua University (China). In this study, pure helium was used as the working gas at a flow rate of 10 SLPM (standard liters per minute). The RF (radio-frequency) power input was $40 \mathrm{~W}$. The distance $(D)$ between the plasma torch nozzle exit and the sample plate was $2 \mathrm{~mm}$ and the plasma jet temperature was below $30^{\circ} \mathrm{C}$. After harvesting spores from a fresh slant medium, 10 
$\mu \mathrm{L}$ of a spore suspension was pipetted onto the stainless steel minidisk. The spores were then exposed to the ARTP jet for different treatment times ranging from $20 \mathrm{~s}$ to $180 \mathrm{~s}$; untreated spores were used as the control. After ARTP mutation, the spore suspension was transferred onto mannitol-soybean (MS) agar at $28^{\circ} \mathrm{C}$ for 72 hour. Single colonies were randomly selected for analysis of antimicrobial activity by dual culture and well diffusion assay. Subsequently, selected mutant strains were cultured in fermentation medium in a shaking incubator $(220 \mathrm{rpm})$ at $28^{\circ} \mathrm{C}$ for $72 \mathrm{~h}$ for wuyiencin analysis.

\subsection{Evaluation of ARTP mutagenesis of $S$. albulus}

Lethality rates of the spores following different treatment periods were evaluated according to the following formula:

Lethality rate $(\%)=(\mathrm{U}-\mathrm{T}) / \mathrm{U} \cdot 100$,

where $U$ is the total colony count of the sample without treatment and $T$ is the total colony count after treatment with ARTP.

Mutation rates of the spores following different treatment periods were calculated according to following formula:

Mutation rate $\left(R_{M}\right)(\%)=M / T \cdot 100$,

where $\mathrm{M}$ is the total colony count of mutants producing different levels of wuyiencin compared with that of the wild-type $S$. albulus CK15 strain.

Positive mutation rates of the spores following different treatment periods were evaluated according to following formula:

Positive mutation rate $\left(R_{P}\right)(\%)=P / M \cdot 100$,

where $\mathrm{P}$ is the total colony count of mutants with increased wuyiencin production compared with that of the wild-type $S$. albulus CK15 strain.

All colony counts were obtained using the colony forming unit (CFU) method on a solid medium.

\subsection{Screening for antimicrobial activity}

All isolates were screened for in vitro antimicrobial activity against pathogenic plant fungi. Screening was performed on the basis of primary and secondary methods. In the dual culture primary screening, $1.1 \mathrm{~cm}^{2}$ of fresh colony was placed in $100 \mathrm{~mL}$ of fermentation medium and cultured in a shaking incubator (220 $\mathrm{rpm}$ ) at $28^{\circ} \mathrm{C}$ for $72 \mathrm{~h}$. The fermentation broth was then collected by filtration. This experiment was repeated on three independent occasions.

This was followed by secondary screening using the agar diffusion method, in which antibacterial effect of the fermentation broth against Rhodotorula rubra was tested by measuring the diameter of 
bacteriostasis circles. These experiments were repeated on five independent occasions.

\subsection{Culture of $S$. albulus in a $5 \mathrm{~L}$ fermenter}

The $S$. albulus strain was inoculated into MS agar medium and incubated at $28{ }^{\circ} \mathrm{C}$ for 7 days, and then approximately $1 \mathrm{~cm}^{2}$ bacterial samples were cultured in $500 \mathrm{~mL}$ flasks containing $50 \mathrm{~mL}$ of the seed medium at $28^{\circ} \mathrm{C}$ and $220 \mathrm{rpm}$ for $24 \mathrm{~h}$. Next, the seed culture was inoculated $(10 \%, \mathrm{v} / \mathrm{v})$ into the $5 \mathrm{~L}$ fermenter containing $3.0 \mathrm{~L}$ of the fermentation medium. Fermentation was carried out at $28^{\circ} \mathrm{C}$ for $64 \mathrm{~h}$ with aeration at $1.0 \mathrm{vvm}$ (air volume/culture volume/ $\mathrm{min}$ ) and fermenter pressure at $0.09 \mathrm{~kg} / \mathrm{cm}^{2}$. The agitation rate was set automatically to maintain dissolved oxygen levels higher than $20 \%$ during the whole fermentation process. The wild-type $S$. albulus CK15 strain was cultured in the same condition.

\subsection{Assay of wuyiencin production}

The concentration of wuyiencin in the fermentation broth was analyzed using the Agilent 1100 highperformance liquid chromatography (HPLC) system equipped with a CAPCELL PAK AQ C18 column (4.6 $\mathrm{mm} \times 250 \mathrm{~mm}$ i.d., $5 \mu \mathrm{m}$ ) at $25^{\circ} \mathrm{C}$. Separation was achieved using a mobile phase consisting of $1.4 \mathrm{~g} / \mathrm{L}$ trichloroacetic acid at a flow rate of $1 \mathrm{~mL} / \mathrm{min}$ and the eluants were detected at a wavelength of $254 \mathrm{~nm}$. The retention time for wuyiencin was 10.5 min under these conditions. The concentration of wuyiencin was calculated by comparing the area of HPLC peaks with the $1000 \mathrm{ppm}$ standard wuyiencin as the control.

\subsection{Detection of the genetic stability of the mutant}

The genetic stability of the mutant was examined by subculture for eight generations. First, the mutant was cultivated on MS medium for 3 to 5 days (the first subculture). Several individual colonies were then selected and cultured on new plates for 3 to 5 days (the second subculture). This procedure was repeated for a total of eight subcultures, after which the wuyiencin yield was detected by HPLC.

\subsection{Statistical analysis}

The data were presented as the means \pm standard deviation (SD) of three independent experiments. Statistical analysis was performed using SPSS 17 software (SPSS Inc). Differences between groups were evaluated by one-way analysis of variance (ANOVA). $P<0.05$ was considered to indicate statistical significance.

\section{Results}

\subsection{Mutation and screening of the mutants}

To determine the optimal ARTP mutagenesis exposure time, the lethality and positive mutation rates were determined after exposure for different periods of time (20,40,60, 80, 100, 120, 150 and $180 \mathrm{~s})$. As shown in Figure 1, the lethality rates of $S$. albulus CK15 increased from $43.75 \%$ after exposure for $40 \mathrm{~s}$ to $83.50 \%$ after exposure for $180 \mathrm{~s}$. Compared with wild-type CK15, fewer colonies were produced after 
ARTP mutagenesis. Following ARTP mutagenesis exposure of the CK15 strain for $180 \mathrm{~s}$, the lethality rate reached $83.50 \%$ and the highest positive mutation rate of $28.00 \%$ was recorded. Therefore, ARTP mutagenesis treatment exposure time applied for this study was determined as $180 \mathrm{~s}$ in this study. In terms of morphological characteristics, some mutants exhibited a slight change in colony color (Figure 2).

After ARTP mutagenesis, 200 mutants were screened using the dual culture antimicrobial method with Rhodotorula rubra as indicator organism. Out of 200 isolates, 56 showed greater antimicrobial activity than CK15 against Rhodotorula rubra (Figure 3). The antibacterial activity of these mutants was then tested in a secondary screen using the agar diffusion method. Three of these mutant strains were selected for further study due to higher antibacterial activity and broad spectrum of activity relative to that of CK15 which experiment were done for five times (Figure 4).

\subsection{Assay of wuyiencin production}

To investigate the cumulative effect of ARTP mutagenesis on wuyiencin production, wuyiencin concentrations in fermentation cultures of all 56 mutant strains were analyzed by HPLC. As shown in Table 1, five strains produced higher concentrations of wuyiencin compared with that of wild-type CK15 strain. Of these, strains M19, M26 and M28 showed an increase in wuyiencin production of more than $10.00 \%$ compared with that of the wild-type CK15 strain. In fermentation cultures, M19 and M26 produced wuyiencin yields of $1430.2 \pm 37.7 \mathrm{U} / \mathrm{mL}$ and $1383.5 \pm 29.6 \mathrm{U} / \mathrm{mL}$, respectively, representing increases of $17.41 \%$ and $13.57 \%$, respectively, compared with that of the wild-type CK15 strain $(1218.09 \pm 74.04 \mathrm{U} / \mathrm{mL})$. The most productive mutant, M28 exhibited a maximum wuyiencin yield of $1442.95 \pm 134.61 \mathrm{U} / \mathrm{mL}$ in flask fermentation, representing an increase of $18.46 \%$ compared with that of CK15 (Figure 5). The M28 produced wuyiencin yield of $1673.84 \pm 127.42 \mathrm{U} / \mathrm{mL}$ in a $5 \mathrm{~L}$ fermenter, representing increases of $21.96 \%$, compared with that of the wild-type CK15 strain $(1372.50 \pm 22.50$ $\mathrm{U} / \mathrm{mL})$.

Table 1

Wuyiencin production by the $S$. albulus wild-type and ARTP mutants

\begin{tabular}{|lll|}
\hline Strain & Wuyiencin $(\mathrm{U} / \mathrm{mL})$ & Increase in production $(\%)$ \\
\hline CK15 & $1218.09 \pm 74.04$ & - \\
\hline M7 & $1238.35 \pm 48.47$ & 1.66 \\
\hline M19 & $1430.20 \pm 37.7$ & 17.41 \\
\hline M26 & $1383.5 \pm 29.6$ & 13.57 \\
\hline M27 & $1303.57 \pm 17.47$ & 7.02 \\
\hline M28 & $1442.95 \pm 134.61$ & 18.46 \\
\hline Data represent the mean \pm standard deviation (SD) from three independent experiments. \\
\hline
\end{tabular}


After cultivating on MS medium under the same condition for eight generations, wuyiencin production of the mutants was detected by HPLC. High wuyiencin productivity of M19, M26 and M28 was maintained through each times (Figure 6).

\section{Discussion}

To meet the requirements for microorganisms and their products, such as secondary metabolites, for agricultural use, high-yield microbial varieties are needed. In the breeding process of high-yield strain of wuyiencin, no stable high-yield strains were obtained through multiple physical and chemical mutagenesis in the previous studies, and many traditional physical and chemical mutagenesis methods are not safe, such as UV mutagenesis and nitrosoguanidine mutagenesis. Although some progress has been developed in molecular breeding of high-yield strains of wuyiencin, this method needs high price and long time. Therefore, a simple and efficient method is needed to breed high-yield strains of wuyiencin.

ARTP is a novel, efficient, safe and environment-friendly mutagenesis tool with a higher mutation rate than those of traditional mutagens (Ottenheim et al. 2018). In this study, ARTP mutagenesis was found to be an efficient strategy to enhance wuyiencin production in S. albulus. Li et al. revealed that ARTP caused alterations in the molecular structure of lipases leading to increased enzyme activity (Li et al. 2011). Zhang et al. revealed that ARTP physical mutagenesis technology in microbial mutation breeding has obvious superiority in spore-forming species with important value for industrial and agricultural applications (Zhang et al. 2014). In this study, S. albulus spores were subjected to ARTP mutagenesis for different periods of times. Our results showed that the lethality rate increased with the duration of the ARTP treatment. Ottenheim et al. (Ottenheim et al. 2018) reported that ARTP treatment time is the main parameter to adjust the exposure of the sample to plasma while keeping all other parameters constant. The death rate curve for Actinomycetes also indicated that effective range for ARTP mutagenesis in these species is between $30 \mathrm{~s}$ and $180 \mathrm{~s}$. In this study, our preliminary experiments showed that ARTP exposure for $180 \mathrm{~s}$ was optimal for generation of mutants. The antimicrobial activity of the mutants was found to be greater than that of the wild-type CK15 strain in dual culture and agar diffusion assays. This indicates that wuyiencin production is greater in fermentation media than in MS media. This is consistent with the report by Khattab et al. (Khattab et al. 2016), which showed that culture conditions play an important role in the production of secondary metabolites by Streptomyces.

In HPLC assay, the increase in wuyiencin yield confirmed that ARTP mutation breeding is an efficient method for generating high-yield mutants. Compared to the initial strain CK15, mutants obtained from ARTP mutagenesis demonstrated a higher wuyiencin production. We also found that optimum ARTP treatment time in this study was similar to other $S$. species under the same ARTP working gas flow rate, ARTP power, and this may be different from other different microorganism. The reason might be the differences in sizes and structures between Streptomyces and other microorganisms. In this research, three mutants M19, M26 and M28 were selected and the genetic stability in terms of wuyiencin content of these mutants was confirmed by subcultures. After the 8th subculture, the specific growth rates of the 
mutants are still high in wuyiencin productivity. Based on genetic stability test, we concluded that the ARTP treatment of $S$. albulus CK15 spores improved wuyiencin production, probably by inducing changes in the metabolic network in mutants. Thus, our results indicate that ARTP treatment is an effective tool for microbial mutation.

\section{Conclusion}

In this study, ARTP was applied to generate mutant wuyiencin-producing strains. This technique showed a strong positive effect on mutation breeding of $S$. albulus. The M28 mutant exhibited the greatest increase in wuyiencin production, with a maximum wuyiencin activity of $1442.95 \pm 134.61 \mathrm{U} / \mathrm{mL}$ in flask culture and $1673.84 \pm 127.42 \mathrm{U} / \mathrm{mL}$ in a $5 \mathrm{~L}$ fermenter, representing an increase of $18.46 \%$ in flask culture and $21.96 \%$ in a $5 \mathrm{~L}$ fermenter compared with that of the wild-type $S$. albulus CK15 strain under the same culture conditions. Furthermore, the stability of the mutant was maintained after eight generations in culture. Thus, it can be concluded that the ARTP treatment of spores improved wuyiencin production, probably by changing the metabolic network in $S$. albulus. Our findings confirm that ARTP mutagenesis is a cost-effective approach for enhancing wuyiencin production and further scale-up fermentation studies are recommended.

\section{Declarations}

\section{Competing interests}

The authors declare that they have no competing interests.

\section{Funding}

This work was supported by the Central Public-interest Scientific Institution Basal Research Fund (Y2020PT04) and the Science and Technology Major Project of Guangxi (AA18242026).

\section{Authors' contributions}

YWT and L.S. carried out the experiment and drafted the manuscript. B.L. and Q.W. participated in the preparation of reagents and data analysis. K.Z. and B.G. designed and supervised the experiment and reviewed the manuscript. All authors read and approved the final manuscript.

\section{Acknowledgments}

We are very grateful to the Department of Chemical Engineering, Tsinghua University, Beijing, China for the use of the ARTP biological breeding system. We also thank the native English speaking scientists of Elixigen Company (Huntington Beach, California) for editing our manuscript.

\section{References}


1. Cao XM, Luo ZS, Zeng WZ, Xu S, Zhao LQ, Zhou JW (2018) Enhanced avermectin production by Streptomyces avermitilis ATCC 31267 using high-throughput screening aided byfluorescenceactivated cell sorting. Appl Microbiol Biotechnol 102(2):703-712

2. Cui ZJ, Zhang KC, She GM, Lin YN, Sun L, Cheng Y, Tan BB (2010) Antimicrobial activity investigation on Wuyiencin fractions of different polarity. Molecules 15(5):3179-3185

3. Fang MY, Jin LH, Zhang C, Tan YY, Jiang PX, Ge N, Li H, Xing P (2013) Rapid mutation of Spirulina platensis by a new mutagenesis system of atmospheric and room temperature plasmas (ARTP) and generation of a mutant library with diverse phenotypes. PLoS ONE 8(10):e77046

4. Ge BB, Cheng Y, Liu Y, Liu BH, Zhang KC (2015) Biological control of Botrytis cinerea on tomato plants using Streptomyces ahygroscopicus strain CK-15. Lett Appl Microbiol 61(6):596-602

5. Gu LS, Tan MZ, Li SH, Zhang T, Zhang QQ, Li CX, Luo XM, Feng JX, Zhao S (2020) ARTP/EMScombined multiple mutagenesis efficiently improved production of raw starch-degrading enzymes in Penicillium oxalicum and characterization of the enzyme-hyperproducing mutant. Biotechnol Biofuels 13(1):187

6. Guo JL, Application Research of Atmospheric Pressure Plasma on Microbial Breeding Physical Mutagenesis Technology (2018). International Conference on Biomedical Engineering, Machinery and Earth Science(BEMES 2018), 2018

7. Khan ST, Komaki H, Motohashi K, Kozone I, Mukai A, Takagi M (2011) Shin-ya, K. Streptomyces associated with a marine sponge Haliclona sp.; biosynthetic genes for secondary metabolites and products. Environ Microbiol 13(2):391-403

8. Khattab Al, Babiker EH, Saeed HA (2016) Streptomyces: isolation, optimization of culture conditions and extraction of secondary metabolites. Int Curr Pharm J 5(3):27-32

9. Kumar AK, Parikh BS, Singh SP, Shah D (2015) Use of combined UV and chemical mutagenesis treatment of Aspergillus terreus D34 for hyper-production of cellulose-degrading enzymes and enzymatic hydrolysis of mild-alkali pretreated rice straw. Bioresources and Bioprocessing 2(1):35

10. Laroussi M (2005) Low Temperature Plasma-Based Sterilization: Overview and State-of-the-Art. Plasma Processes Polym 2(5):391-400

11. Li G, Li HP, Wang LY, Wang S, Zhao HX, Sun WT, Xing XH, Bao CY (2008) Genetic effects of radiofrequency, atmospheric-pressure glow discharges with helium. Appl Phys Lett 92(22):221504

12. Li HG, Luo W, Wang Q, Yu XB (2014) Direct fermentation of gelatinized cassava starch to acetone, butanol, and ethanol using Clostridium acetobutylicum mutant obtained by atmospheric and room temperature plasma. Appl Biochem Biotechnol 172(7):3330-3341

13. Li HP, Wang LY, Li G, Jin LH, Le PS, Zhao HX, Xing XH, Bao CY (2011) Manipulation of lipase activity by the helium radio-frequency, atmospheric-pressure glow discharge plasma jet. Plasma Processes Polym 8(3):224-229

14. Liu KY, Fang H, Cui FJ, Nyabako BA, Tao TL, Zan XY, Chen HY, Sun WJ (2020) ARTP mutation and adaptive laboratory evolution improve probiotic performance of Bacillus coagulans. Appl Microbiol Biotechnol 104(14):6363-6373 
15. Liu YY, Ryu H, Ge BB, Pan GH, Sun L, Park K, Zhang KC (2014) Improvement of Wuyiencin biosynthesis in Streptomyces wuyiensis CK-15 by identification of a key regulator, WysR. J Microbiol Biotechnol 24(12):1644-1653

16. Lu Y, Wang LY, Ma K, Li G, Zhang C, Zhao HX, Lai QH, Li HP, Xing XH (2011) Characteristics of hydrogen production of an Enterobacter aerogenes mutant generated by a new atmospheric and room temperature plasma (ARTP). Biochem Eng J 55(1):17-22

17. Mohanraj G, Sekar T (2013) Isolation and screening of actinomycetes from marine sediments for their potential to produce antimicrobials. Int J Life Sci Pharma Res 2(12):115-126

18. Ōmura S, Ikeda H, Ishikawa J, Hanamoto A, Takahashi C, Shinose M, Takahashi Y, Horikawa H, Nakazawa H, Osonoe T, Kikuchi H, Shiba T, Sakaki Y, Hattori M (2001) Genome sequence of an industrial microorganism Streptomyces avermitilis: deducing the ability of producing secondary metabolites. Proceedings of the National Academy of Sciences. 98 (21):12215-12220

19. Ottenheim C, Nawrath M, Wu JC (2018) Microbial mutagenesis by atmospheric and roomtemperature plasma (ARTP): the latest development. Bioresources and Bioprocessing 5(1):12

20. Patzer SI, Braun V (2010) Gene cluster involved in the biosynthesis of griseobactin, a catecholpeptide siderophore of Streptomyces sp. ATCC 700974. J Bacteriol 192(2):426-435

21. Wang Q, Feng LR, Wei L, Li HG, Wang L, Zhou Y, Yu XB (2014) Mutation breeding of lycopeneproducing strain Blakeslea trispora by a novel atmospheric and room temperature plasma (ARTP). Appl Biochem Biotechnol 174(1):452-460

22. Qiu L, Nie SX, Hu SJ, Wang SJ, Wang JJ, Guo K (2021) Screening of Beauveria bassiana with high biocontrol potential based on ARTP mutagenesis and high-throughput FACS. Pestic Biochem Physiol 171:104732

23. Ren F, Chen L, Tong QY (2017) Highly improved acarbose production of Actinomyces through the combination of ARTP and penicillin susceptible mutant screening. World J Microbiol Biotechnol 33(1):16

24. Sivaramakrishnan R, Incharoensakdi A (2017) Enhancement of lipid production in Scenedesmus sp. by UV mutagenesis and hydrogen peroxide treatment. Bioresour Technol 235:366-370

25. Tan J, Chu J, Wang YH, Zhuang YP, Zhang SL (2014) High-throughput system for screening of Monascus purpureus high-yield strain in pigment production. Bioresources and Bioprocessing $1(1): 16$

26. Wang HY, Zhang J, Zhang YJ, Zhang B, Liu CX, He HR, Wang XJ, Xiang WS (2014) Combined application of plasma mutagenesis and gene engineering leads to 5-oxomilbemycins A3/A4 as main components from Streptomyces bingchenggensis. Appl Microbiol Biotechnol 98(23):9703-9712

27. Wang LY, Zhao HX, He D, Wu YN, Jin LH, Li G, Su N, Li HP, Xing XH (2020) Insights into the molecularlevel effects of atmospheric and room-temperature plasma on mononucleotides and single-stranded homo- and hetero-oligonucleotides. Sci Rep 10(1):14298

28. Wang LY, Huang ZL, Li G, Zhao HX, Xing XH, Sun WT, Li HP, Gou ZX, Bao CY (2010) Novel mutation breeding method for Streptomyces avermitilis using an atmospheric pressure glow discharge 
plasma. J Appl Microbiol 108(3):851-858

29. Xu F, Jin HL, Li HM, Tao L, Wang JP, Lv J, Chen S (2012) Genome shuffling of Trichoderma viride for enhanced cellulase production. Ann Microbiol 62(2):509-515

30. Zhang CL, Shen HW, Zhang XB, Yu X, Wang H, Xiao S, Wang JH, Zhao ZB (2016) K. Combined mutagenesis of Rhodosporidium toruloides for improved production of carotenoids and lipids. Biotechnol Lett 38(10):1733-1738

31. Zhang X, Zhang XF, Li HP, Wang LY, Zhang C, Xing XH, Bao CY (2014) Atmospheric and room temperature plasma (ARTP) as a new powerful mutagenesis tool. Appl Microbiol Biotechnol 98(12):5387-5396

\section{Figures}

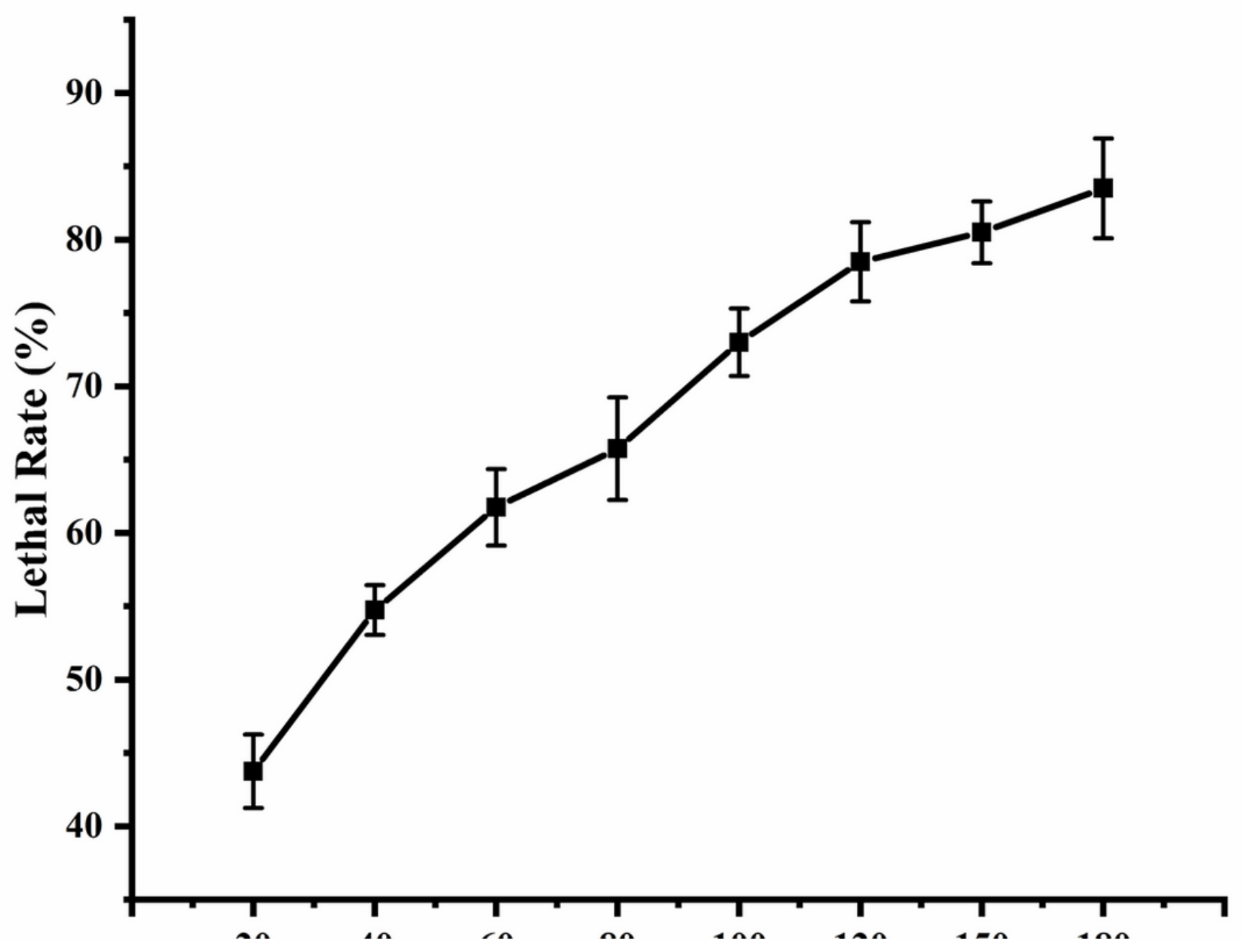

Figure 1 
Lethality rate of $S$. albulus CK15 after ARTP mutagenesis.

\section{Figure 2}

Effect of ARTP mutagenesis on S. albulus CK15.

(a) Morphology of colonies cultured 3 days after mutagenesis with different ARTP exposure times (20 $\mathrm{CFU})$.

(b) Morphology of CK15 and mutants M19, M26 and M28 cultured for 14 days.

\section{Figure 3}

Agar diffusion assay of the antimicrobial activity of CK15 mutant strains generated by ARTP mutagenesis for 180 s. 1-56 means M1-M56, respectively.

\section{Figure 4}

Agar diffusion assay of the antimicrobial activity of CK15 and three selected mutants.

Values are means \pm S.E.M of three independent experiments.

\section{Figure 5}

Effect of ARTP on wuyiencin production.

(a) HPLC analysis of wuyiencin production levels in S. albulus CK15. (b) HPLC analysis of wuyiencin production levels in M28. (c) HPLC analysis of standard wuyiencin. (d) HPLC analysis of wuyiencin production levels by M28 and CK15, black peak indicates wuyiencin production levels by CK15, pink peak indicates wuyiencin production levels by $\mathrm{M} 28$.

\section{Figure 6}

The genetic stability of $S$. albulus CK15 and three selected mutants. Values are means \pm S.E.M of three independent experiments. 